\title{
Inverse problem for a class of Sturm-Liouville operator with spectral parameter in boundary condition
}

\author{
Khanlar R Mamedov ${ }^{*}$ and F Ayca Cetinkaya
}

\section{*Correspondence:}

hanlar@mersin.edu.tr

Department of Mathematics,

Science and Letters Faculty, Mersin

University, Mersin, 33343, Turkey

\begin{abstract}
This work aims to examine a Sturm-Liouville operator with a piece-wise continuous coefficient and a spectral parameter in boundary condition. The orthogonality of the eigenfunctions, realness and simplicity of the eigenvalues are investigated. The asymptotic formula of the eigenvalues is found, and the resolvent operator is constructed. It is shown that the eigenfunctions form a complete system and the expansion formula with respect to eigenfunctions is obtained. Also, the evolution of the Weyl solution and Weyl function is discussed. Uniqueness theorems for the solution of the inverse problem with Weyl function and spectral data are proved. MSC: 34L10; 34L40; 34A55
\end{abstract}

Keywords: Sturm-Liouville operator; expansion formula; inverse problem; Weyl function

\section{Introduction}

In recent years, there has been a growing interest in physical applications of boundary value problems with a spectral parameter, contained in the boundary conditions. The relationship between diffusion processes and Sturm-Liouville problem with eigen-parameter in the boundary conditions has been shown in [1]. Another example of this relationship between the same problem and the wave equation has been examined in [2, 3]. SturmLiouville problems with a discontinuous coefficient arise upon non-homogeneous material properties.

In a finite interval, inverse problems for the Sturm-Liouville operator with spectral parameter, contained in the boundary conditions, have been investigated, and the uniqueness of the solution of these problems has been shown in [4-9]. The inverse problem has been analyzed by zeros of the eigenfunctions in [6], by numerical methods in [9] and by two spectra, consisting of sequences of eigenvalues and the normed constants in [10]. In $[11,12]$, eigenvalue-dependent inverse problem with the discontinuities inside the interval was examined by the Weyl function. In a finite interval, discontinuous and no eigenvalue parameter containing direct problem and inverse problem with the Weyl function were discussed in $[13,14]$. The similar problem was investigated in the half line by scattering data in $[15,16]$.

We consider the boundary value problem

$$
-y^{\prime \prime}+q(x) y=\lambda^{2} \rho(x) y,
$$

(0) 2013 Mamedov and Cetinkaya; licensee Springer. This is an Open Access article distributed under the terms of the Creative Commons Attribution License (http://creativecommons.org/licenses/by/2.0), which permits unrestricted use, distribution, and reproduction in any medium, provided the original work is properly cited. 


$$
\begin{aligned}
& U_{1}(y):=y(0)=0, \\
& U_{2}(y):=\lambda^{2}\left[\beta_{1} y^{\prime}(\pi)+\beta_{2} y(\pi)\right]+\alpha_{1} y(\pi)+\alpha_{2} y^{\prime}(\pi)=0,
\end{aligned}
$$

where $q(x) \in L_{2}(0, \pi)$ is a real valued function, $\lambda$ is a complex parameter, $\alpha_{1}, \alpha_{2}, \beta_{1}, \beta_{2}$ are real numbers and

$$
\rho(x)= \begin{cases}1, & 0 \leq x<a, \\ \alpha^{2}, & a<x \leq \pi\end{cases}
$$

where $0<\alpha \neq 1$.

\section{Special solutions}

Let $\varphi(x, \lambda)$ and $\psi(x, \lambda)$ be the solutions of equation (1) satisfying the initial conditions

$$
\begin{aligned}
& \varphi(0, \lambda)=0, \quad \varphi^{\prime}(0, \lambda)=1, \\
& \psi(\pi, \lambda)=-\lambda^{2} \beta_{1}-\alpha_{2}, \quad \psi^{\prime}(\pi, \lambda)=\lambda^{2} \beta_{2}+\alpha_{1} .
\end{aligned}
$$

For the solution of equation (1), the following integral representation is obtained in [13] for all $\lambda$ :

$$
\begin{aligned}
e(x, \lambda)= & \frac{1}{2}\left(1+\frac{1}{\sqrt{\rho(x)}}\right) e^{i \lambda \mu^{+}(x)}+\frac{1}{2}\left(1-\frac{1}{\sqrt{\rho(x)}}\right) e^{i \lambda \mu^{-}(x)} \\
& +\int_{-\mu^{+}(x)}^{\mu^{+}(x)} K(x, t) e^{i \lambda t} d t,
\end{aligned}
$$

where $K(x, \cdot) \in L_{1}\left(-\mu^{+}(x), \mu^{+}(x)\right)$.

The kernel $K(x, t)$ has the partial derivative $K_{x}^{\prime}$ belonging to the space $L_{1}\left(-\mu^{+}(x), \mu^{+}(x)\right)$ for every $x \in[0, \pi]$, and the properties below hold:

$$
\begin{aligned}
& \frac{d}{d x} K\left(x, \mu^{+}(x)\right)=\frac{1}{4 \sqrt{\rho(x)}}\left(1+\frac{1}{\sqrt{\rho(x)}}\right) q(x) \\
& \frac{d}{d x}\left\{K\left(x, \mu^{-}(x)+0\right)-K\left(x, \mu^{-}(x)-0\right)\right\}=\frac{1}{4 \sqrt{\rho(x)}}\left(1-\frac{1}{\sqrt{\rho(x)}}\right) q(x), \\
& K\left(x,-\mu^{+}(x)\right)=0 .
\end{aligned}
$$

Moreover, if $q(x)$ is differentiable, then the following are valid

$$
\begin{aligned}
& \rho(x) K_{t t}^{\prime \prime}-K_{x x}^{\prime \prime}+q(x) K=0, \quad|t|<\mu^{+}(x) \\
& \int_{-\mu^{+}(x)}^{\mu^{+}(x)}|K(x, t) d t| \leq C\left(\exp \left\{\int_{0}^{x}|q(t)| d t\right\}-1\right), \quad 0<C=\text { const. }
\end{aligned}
$$

Using the representation of the solution $e(x, \lambda)$ and formula

$$
\varphi(x, \lambda)=\frac{e(x, \lambda)-e(x,-\lambda)}{2 i \lambda},
$$


we obtain the integral representation of the solution $\varphi(x, \lambda)$

$$
\varphi(x, \lambda)=\varphi_{0}(x, \lambda)+\int_{0}^{\mu^{+}(x)} A(x, t) \frac{\sin \lambda t}{\lambda} d t
$$

where $A(x, t)=K(x, t)-K(x,-t)$. The kernel $A(x, t)$ can be represented with the coefficients $\rho(x)$ and $q(x)$

$$
\begin{aligned}
& \frac{d}{d x} A\left(x, \mu^{+}(x)\right)=\frac{1}{4 \sqrt{\rho(x)}}\left(1+\frac{1}{\sqrt{\rho(x)}}\right) q(x), \\
& \frac{d}{d x}\left\{A\left(x, \mu^{-}(x)+0\right)-A\left(x, \mu^{-}(x)-0\right)\right\}=\frac{1}{4 \sqrt{\rho(x)}}\left(1-\frac{1}{\sqrt{\rho(x)}}\right) q(x) .
\end{aligned}
$$

With the help of equation (6), we have a representation for the function $\psi(x, \lambda)$

$$
\begin{aligned}
\psi(x, \lambda)= & -\frac{1}{2}\left(1+\frac{\alpha}{\sqrt{\rho(x)}}\right) \frac{\sin \lambda\left(\mu^{+}(\pi)-\mu^{+}(x)\right)}{\alpha \lambda} \\
& -\frac{1}{2}\left(1-\frac{\alpha}{\sqrt{\rho(x)}}\right) \frac{\sin \lambda\left(\mu^{+}(\pi)-\mu^{-}(x)\right)}{\alpha \lambda} \\
& +\int_{0}^{\mu^{+}(\pi)-\mu^{+}(x)} \tilde{A}(x, t) \frac{\sin \lambda t}{\lambda} d t,
\end{aligned}
$$

where $\tilde{A}(x, t)$ is a real function.

Denote

$$
\Delta(\lambda):=\langle\varphi(x, \lambda), \psi(x, \lambda)\rangle=\varphi(x, \lambda) \psi^{\prime}(x, \lambda)-\varphi^{\prime}(x, \lambda) \psi(x, \lambda),
$$

which is independent of $x \in[0, \pi]$. Substituting $x=0$ and $x=\pi$ into (8), we get

$$
\Delta(\lambda)=U_{2}(\varphi)=-U_{1}(\psi)
$$

$\Delta(\lambda)$ is an entire function of $\lambda$ and is called the characteristic function of the boundary value problem (1)-(3).

\section{Some spectral properties}

Lemma 1 The square values of the roots $\left(\lambda_{n}\right)_{n=0}^{\infty}$ of the characteristic function coincide with the eigenvalues of the boundary value problem (1)-(3), and for every $\lambda_{n}$, there exists a sequence $\left(k_{n}\right)$ such that

$$
\psi\left(x, \lambda_{n}\right)=k_{n} \varphi\left(x, \lambda_{n}\right) \quad\left(k_{n} \neq 0\right)
$$

where $\psi\left(x, \lambda_{n}\right)$ and $\varphi\left(x, \lambda_{n}\right)$ are the eigenfunctions of the boundary value problem (1)-(3), corresponding to the eigenvalue $\lambda_{n}$.

Proof The proof can be done in a similar way to [8]. Indeed, let us assume that $\lambda_{0}$ is an eigenvalue of the function $\Delta(\lambda)$. Then

$$
\Delta(\lambda)=\left|\begin{array}{ll}
\varphi\left(x, \lambda_{0}\right) & \psi\left(x, \lambda_{0}\right) \\
\varphi^{\prime}\left(x, \lambda_{0}\right) & \psi^{\prime}\left(x, \lambda_{0}\right)
\end{array}\right|=0
$$


holds, i.e., the functions $\varphi\left(x, \lambda_{0}\right)$ and $\psi\left(x, \lambda_{0}\right)$ are linearly dependent $\psi\left(x, \lambda_{0}\right)=k_{n} \varphi\left(x, \lambda_{0}\right)$ $\left(k_{n}=\right.$ const.), and they satisfy the boundary conditions (2), (3). Hence $\lambda_{0}^{2}$ is an eigenvalue, $\psi\left(x, \lambda_{0}\right)$ and $\varphi\left(x, \lambda_{0}\right)$ are eigenfunctions, related to this eigenvalue. Conversely, let $\lambda_{0}^{2}$ be an eigenvalue of the operator $A$, and let $y_{0}\left(x, \lambda_{0}\right), y_{0}\left(x,-\lambda_{0}\right)$ be the corresponding eigenfunctions. Then the boundary conditions (2), (3) hold both for the eigenfunctions $y_{0}\left(x, \lambda_{0}\right)$ and $y_{0}\left(x,-\lambda_{0}\right)$. Additionally, if the functions $y_{0}\left(x, \lambda_{0}\right)$ and $y_{0}\left(x,-\lambda_{0}\right)$ satisfy the conditions $y^{\prime}\left(0, \lambda_{0}\right)=1, y^{\prime}\left(0,-\lambda_{0}\right)=1$, then $y_{0}\left(x, \lambda_{0}\right) \equiv \varphi\left(x, \lambda_{0}\right), y_{0}\left(x,-\lambda_{0}\right) \equiv \varphi\left(x,-\lambda_{0}\right)$. According to boundary conditions (2), (3), we have

$$
\begin{aligned}
& \Delta\left(\lambda_{0}\right)=U_{2}\left(\varphi\left(\pi, \lambda_{0}\right)\right)=U_{2}\left(y_{0}\left(\pi, \lambda_{0}\right)\right)=0, \\
& \Delta\left(-\lambda_{0}\right)=U_{2}\left(\varphi\left(\pi,-\lambda_{0}\right)\right)=U_{2}\left(y_{0}\left(\pi,-\lambda_{0}\right)\right)=0 .
\end{aligned}
$$

Similarly, if we assume that $y_{0}^{\prime}\left(\pi, \lambda_{0}\right)=\lambda_{0}^{2} \beta_{2}+\alpha_{1}, y_{0}^{\prime}\left(\pi,-\lambda_{0}\right)=\lambda_{0}^{2} \beta_{2}+\alpha_{1}$, then $y_{0}\left(x, \lambda_{0}\right) \equiv$ $\psi\left(x, \lambda_{0}\right), y_{0}\left(x,-\lambda_{0}\right) \equiv \psi\left(x,-\lambda_{0}\right)$. Again from the boundary conditions (2), (3), it is obvious that

$$
\begin{aligned}
& \Delta\left(\lambda_{0}\right)=-U_{1}\left(\psi\left(0, \lambda_{0}\right)\right)=-U_{1}\left(y_{0}\left(0, \lambda_{0}\right)\right)=0, \\
& \Delta\left(-\lambda_{0}\right)=-U_{1}\left(\psi\left(0,-\lambda_{0}\right)\right)=-U_{1}\left(y_{0}\left(0,-\lambda_{0}\right)\right)=0 .
\end{aligned}
$$

Therefore, we have proved that for each eigenvalue $\lambda_{0}^{2}$, there exists only one (up to a multiplicative constant) eigenfunction.

In the Hilbert space $H_{\rho}=L_{2, \rho}(0, \pi) \oplus \mathbb{C}$ an inner product defined by

$$
(F, G):=\int_{0}^{\pi} F_{1}(x) \overline{G_{1}(x)} \rho(x) d x+\frac{F_{2} \overline{G_{2}}}{\chi},
$$

where

$$
F=\left(\begin{array}{c}
F_{1}(x) \\
F_{2}
\end{array}\right) \in H_{\rho}, \quad G=\left(\begin{array}{c}
G_{1}(x) \\
G_{2}
\end{array}\right) \in H_{\rho}, \quad \chi:=\alpha_{1} \beta_{1}-\alpha_{2} \beta_{2}>0 .
$$

Let us define

$$
A(F):=\left(\begin{array}{l}
-F_{1}^{\prime \prime}(x)+q(x) F_{1}(x) \\
\alpha_{1} F_{1}(\pi)+\alpha_{2} F_{1}^{\prime}(\pi)
\end{array}\right)
$$

with

$$
D(A)=\left\{\begin{array}{c}
F \in H_{\rho}: F_{1}(x), F_{1}^{\prime}(x) \in A C[0, \pi], l F_{1} \in L_{2, \rho}(0, \pi) \\
F_{1}(0)=0, F_{2}=\beta_{1} F_{1}^{\prime}(\pi)+\beta_{2} F_{1}(\pi)
\end{array}\right\},
$$

where

$$
l\left(F_{1}\right)=\frac{1}{\rho(x)}\left\{-F_{1}^{\prime \prime}+q(x) F_{1}\right\} .
$$

The boundary value problem (1)-(3) is equivalent to the equation $A Y=\lambda^{2} Y$. 
The eigenfunctions of operator $A$ are in the form of

$$
\Phi\left(x, \lambda_{n}\right)=\Phi_{n}:=\left(\begin{array}{c}
\varphi\left(x, \lambda_{n}\right) \\
\beta_{1} \varphi^{\prime}\left(\pi, \lambda_{n}\right)+\beta_{2} \varphi\left(\pi, \lambda_{n}\right)
\end{array}\right) .
$$

Lemma 2 The eigenfunctions $\Phi_{1}$ and $\Phi_{2}$, corresponding to different eigenvalues $\lambda_{1} \neq \lambda_{2}$, are orthogonal.

Proof Since $\Phi_{1}$ and $\Phi_{2}$ are the solutions of the boundary value problem (1)-(3), the equations below are valid

$$
\begin{aligned}
& -\Phi^{\prime \prime}\left(x, \lambda_{1}\right)+q(x) \Phi\left(x, \lambda_{1}\right)=\lambda_{1}^{2} \rho(x) \Phi\left(x, \lambda_{1}\right) \\
& -\Phi^{\prime \prime}\left(x, \lambda_{2}\right)+q(x) \Phi\left(x, \lambda_{2}\right)=\lambda_{2}^{2} \rho(x) \Phi\left(x, \lambda_{2}\right) .
\end{aligned}
$$

Multiplying the first equation by $\Phi\left(x, \lambda_{2}\right)$ and the second equation by $-\Phi\left(x, \lambda_{1}\right)$ and adding together, we have

$$
\frac{d}{d x}\left\{\Phi\left(x, \lambda_{1}\right) \Phi^{\prime}\left(x, \lambda_{2}\right), \Phi^{\prime}\left(x, \lambda_{1}\right) \Phi\left(x, \lambda_{2}\right)\right\}=\left(\lambda_{1}^{2}-\lambda_{2}^{2}\right) \rho(x) \Phi\left(x, \lambda_{1}\right) \Phi\left(x, \lambda_{2}\right) .
$$

Integrating it from 0 to $\pi$, and using the boundary condition (3), we obtain

$$
\begin{aligned}
& \left(\lambda_{1}^{2}-\lambda_{2}^{2}\right)\left[\int_{0}^{\pi} \Phi\left(x, \lambda_{1}\right) \Phi\left(x, \lambda_{2}\right) \rho(x) d x\right] \\
& \quad+\left(\lambda_{1}^{2}-\lambda_{2}^{2}\right)\left[\frac{1}{k}\left(\beta_{1} \varphi^{\prime}\left(x, \lambda_{1}\right)+\beta_{2} \varphi\left(x, \lambda_{1}\right)\right)\left(\beta_{1} \varphi^{\prime}\left(x, \lambda_{2}\right)+\beta_{2} \varphi\left(x, \lambda_{2}\right)\right)\right]=0 .
\end{aligned}
$$

Since $\lambda_{1} \neq \lambda_{2}$, the lemma is proved.

Corollary 3 The eigenvalues of the boundary value problem (1)-(3) are real.

The values

$$
\gamma_{n}=\int_{0}^{\pi} \varphi^{2}\left(x, \lambda_{n}\right) d x+\frac{\left(\beta_{1} \varphi^{\prime}\left(\pi, \lambda_{n}\right)+\beta_{2} \varphi\left(\pi, \lambda_{n}\right)\right)^{2}}{\chi}
$$

are called the norming constants of the boundary value problem (1)-(3).

Now, let us agree to denote differentiation with respect to $\lambda$ with a dot $\dot{\Delta}=\frac{d}{d \lambda} \Delta(\dot{\Delta} \neq 0)$.

Lemma 4 The following equality holds

$$
\dot{\Delta}\left(\lambda_{n}\right)=2 \lambda_{n} k_{n} \gamma_{n}
$$

Proof Since

$$
-\varphi^{\prime \prime}\left(x, \lambda_{n}\right)+q(x) \varphi\left(x, \lambda_{n}\right)=\lambda_{n}^{2} \varphi\left(x, \lambda_{n}\right), \quad-\psi^{\prime \prime}(x, \lambda)+q(x) \psi(x, \lambda)=\lambda^{2} \psi(x, \lambda),
$$

we get

$$
\frac{d}{d x} \Delta(\lambda)=\left(\lambda_{n}^{2}-\lambda^{2}\right) \varphi\left(x, \lambda_{n}\right) \psi(x, \lambda)
$$


With the help of (2) and (3),

$$
-\Delta(\lambda)=\left(\lambda_{n}-\lambda\right)\left(\lambda_{n}+\lambda\right) k_{n}\left[\int_{0}^{\pi} \varphi^{2}\left(x, \lambda_{n}\right) \rho(x) d x+\chi\right] .
$$

Taking into consideration (9) and (10), for $\lambda \rightarrow \lambda_{n}$, we arrive (11).

Corollary 5 All zeros of $\Delta(\lambda)$ are simple, i.e., $\dot{\Delta}\left(\lambda_{n}\right) \neq 0$.

\section{Asymptotic formulas of eigenvalues}

Let $\varphi_{0}(x, \lambda)$ be the solution of equation (1) satisfying the initial conditions (4) when $q(x) \equiv$ 0

$$
\varphi_{0}(x, \lambda)=\frac{1}{2}\left(1+\frac{1}{\sqrt{\rho(x)}}\right) \frac{\sin \lambda\left(\mu^{+}(x)\right)}{\lambda}+\frac{1}{2}\left(1-\frac{1}{\sqrt{\rho(x)}}\right) \frac{\sin \lambda\left(\mu^{-}(x)\right)}{\lambda} .
$$

The eigenvalues $\lambda_{n}^{0}(n=0, \pm 1, \pm 2, \ldots)$ of the boundary value problem (1)-(3) when $q(x) \equiv$ 0 can be found using the equation

$$
\Delta_{0}(\lambda)=\lambda^{2}\left[\beta_{1} \varphi_{0}^{\prime}(\pi, \lambda)+\beta_{2} \varphi_{0}(\pi, \lambda)\right]+\alpha_{1} \varphi_{0}(\pi, \lambda)+\alpha_{2} \varphi_{0}^{\prime}(\pi, \lambda)=0
$$

from [17] (see also [18]) and can be represented in the following way

$$
\lambda_{n}^{0}=\frac{n \pi}{\mu^{+}(\pi)}+h_{n}, \quad n=0, \pm 1, \pm 2, \ldots
$$

where $\sup _{n}\left|h_{n}\right|=h<+\infty$.

Lemma 6 Roots $\lambda_{n}^{0}$ of the function $\Delta_{0}(\lambda)$ are separated, i.e.,

$$
\inf _{n \neq k}\left|\lambda_{n}^{0}-\lambda_{k}^{0}\right|=\tau>0
$$

Proof Assume the contrary. Then there are sequences $\left(\lambda_{k}^{0 \prime}\right),\left(\lambda_{k}^{0 \prime \prime}\right)$ of zeros of functions $\lambda^{2}\left[\beta_{1} y^{\prime}(\pi)+\beta_{2} y(\pi)\right]+\alpha_{1} y(\pi)+\alpha_{1} y^{\prime}(\pi)=0$ such that

$$
\begin{aligned}
& \lambda_{k}^{0 \prime} \neq \lambda_{k}^{0 \prime \prime}, \quad \lambda_{k}^{0 \prime} \rightarrow \infty, \quad \lambda_{k}^{0 \prime \prime} \rightarrow \infty, \\
& \lim _{k \rightarrow \infty}\left(\lambda_{k}^{0 \prime}-\lambda_{k}^{0 \prime \prime}\right)=0 .
\end{aligned}
$$

Since the eigenfunctions $\varphi_{0}\left(x, \lambda_{k}^{0 \prime}\right), \varphi_{0}\left(x, \lambda_{k}^{0 \prime \prime}\right)$ are orthogonal, we have

$$
\begin{aligned}
0= & \lambda_{k}^{0 \prime} \lambda_{k}^{0 \prime \prime} \int_{0}^{\pi} \rho(x) \varphi_{0}\left(x, \lambda_{k}^{0 \prime}\right) \varphi_{0}\left(x, \lambda_{k}^{0 \prime \prime}\right) d x \\
= & \int_{0}^{\pi} \rho(x) \lambda_{k}^{0 \prime} \varphi_{0}\left(x, \lambda_{k}^{0 \prime}\right)\left[\lambda_{k}^{0 \prime \prime} \varphi_{0}\left(x, \lambda_{k}^{0 \prime \prime}\right)-\lambda_{k}^{0 \prime} \varphi_{0}\left(x, \lambda_{k}^{0 \prime}\right)\right] d x \\
& +\int_{0}^{\pi} \rho(x)\left(\lambda_{k}^{0 \prime} \varphi_{0}\left(x, \lambda_{k}^{0 \prime}\right)\right)^{2} d x \\
= & I_{k}+\int_{0}^{\pi} \rho(x)\left(\lambda_{k}^{0 \prime} \varphi_{0}\left(x, \lambda_{k}^{0 \prime}\right)\right)^{2} d x
\end{aligned}
$$




$$
\begin{aligned}
& \geq I_{k}+\int_{0}^{a} \rho(x)\left(\lambda_{k}^{0 \prime} \varphi_{0}\left(x, \lambda_{k}^{0 \prime}\right)\right)^{2} d x=I_{k}+\int_{0}^{a} \sin ^{2} \lambda_{k}^{0 \prime} x d x \\
& =I_{k}+\frac{a}{2}-\frac{\sin 2 \lambda_{k}^{0} a}{4 \lambda_{k}^{0}}
\end{aligned}
$$

where

$$
I_{k}=\int_{0}^{\pi} \rho(x) \lambda_{k}^{0 \prime} \varphi_{0}\left(x, \lambda_{k}^{0 \prime}\right)\left[\lambda_{k}^{0 \prime \prime} \varphi_{0}\left(x, \lambda_{k}^{0 \prime \prime}\right)-\lambda_{k}^{0 \prime} \varphi_{0}\left(x, \lambda_{k}^{0 \prime}\right)\right] d x
$$

Thus,

$$
0 \geq I_{k}+\frac{a}{2}-\frac{\sin 2 \lambda_{k}^{0} a}{4 \lambda_{k}^{0}} .
$$

Let us prove that $I_{k} \rightarrow 0$ as $k \rightarrow \infty$. In fact, (12) implies the following estimate

$$
\left|\lambda_{k}^{0 \prime} \varphi\left(x, \lambda_{k}^{0 \prime}\right)-\lambda_{k}^{0 \prime \prime} \varphi\left(x, \lambda_{k}^{0 \prime \prime}\right)\right| \leq C\left|\lambda_{k}^{0 \prime}-\lambda_{k}^{0 \prime \prime}\right|
$$

Consequently, $\lim _{k \rightarrow \infty}\left[\lambda_{k}^{0 \prime} \varphi\left(x, \lambda_{k}^{0 \prime}\right)-\lambda_{k}^{0 \prime \prime} \varphi\left(x, \lambda_{k}^{0 \prime \prime}\right)\right]=0$ holds uniformly on $x \in[0, \pi]$. Now, passing to the limit in equality (14), as $k \rightarrow \infty$, we have $0 \geq \frac{a}{2}$. This is a contradiction, and it proves the validity of lemma's statement.

Lemma 7 The eigenvalues of the boundary value problem (1)-(3) are in the form of

$$
\lambda_{n}=\lambda_{n}^{0}+\frac{d_{n}}{\lambda_{n}^{0}}+\frac{\eta_{n}}{n}, \quad \lambda_{n} \geq 0,
$$

where $d_{n}$ is a bounded sequence

$$
\begin{aligned}
d_{n}= & \frac{1}{4 \lambda_{n}^{0} \dot{\Delta}_{0}\left(\lambda_{n}^{0}\right)}\left\{-\int_{0}^{\pi} \frac{1}{\sqrt{\rho(t)}}\left(1-\frac{1}{\sqrt{\rho(t)}}\right) q(t) \cos \left(\lambda_{n}^{0} \mu^{-}(\pi)\right) d t\right\} \\
& +\frac{1}{4 \lambda_{n}^{0} \dot{\Delta}_{0}\left(\lambda_{n}^{0}\right)}\left\{\int_{0}^{\pi} \frac{1}{\sqrt{\rho(t)}}\left(1+\frac{1}{\sqrt{\rho(t)}}\right) q(t) \cos \left(\lambda_{n}^{0} \mu^{+}(\pi)\right) d t\right\},
\end{aligned}
$$

and $\left\{\eta_{n}\right\} \in l_{2}$.

Proof From (6), it follows that

$$
\Delta(\lambda)=\Delta_{0}(\lambda)+\int_{0}^{\mu^{+}(\pi)} A(\pi, t) \frac{\sin \lambda t}{\lambda} d t .
$$

Lemma 1.3.1 in [19] and from [17], we get

$$
\Delta(\lambda)-\Delta_{0}(\lambda)=O\left(\frac{1}{|\lambda|}\right) e^{|\operatorname{Im} \lambda| \mu^{+}(\pi)}, \quad|\lambda| \rightarrow \infty .
$$

Therefore, for sufficiently large $n$, on the contours

$$
\Gamma_{n}=\left\{\lambda:|\lambda|=\left|\lambda_{n}^{0}\right|+\frac{\tau}{2}\right\},
$$


we have

$$
\left|\Delta(\lambda)-\Delta_{0}(\lambda)\right|<\left|\Delta_{0}(\lambda)\right|
$$

By the Rouche theorem, we obtain that the number of zeros of the function $\{\Delta(\lambda)-$ $\left.\Delta_{0}(\lambda)\right\}+\Delta_{0}(\lambda)=\Delta(\lambda)$ inside the contour $\Gamma_{n}$ coincides with the number of zeros of the function $\Delta_{0}(\lambda)$. Furthermore, applying the Rouche theorem to the circle $\sigma_{n}(\delta)=\{\lambda$ : $\left.\left|\lambda-\lambda_{n}^{0}\right| \leq \delta\right\}$, we get that, for sufficiently large $n$ there exists only one zero $\lambda_{n}$ of the function $\Delta(\lambda)$ in $\sigma_{n}(\delta)$. Owing to the arbitrariness of $\delta>0$ we have

$$
\lambda_{n}=\lambda_{n}^{0}+\epsilon_{n}, \quad \epsilon_{n}=o(1), n \rightarrow \infty .
$$

Substituting (18) into (16), we get

$$
\Delta\left(\lambda_{n}^{0}+\epsilon_{n}\right)=\Delta_{0}\left(\lambda_{n}^{0}+\epsilon_{n}\right)+\int_{0}^{\mu^{+}(\pi)} A(\pi, t) \frac{\sin \left(\lambda_{n}^{0}+\epsilon_{n}\right)}{\lambda_{n}^{0}+\epsilon_{n}} d t=0 .
$$

Hence, as $n \rightarrow \infty$, taking into account the equality $\Delta_{0}\left(\lambda_{n}^{0}\right)=0$ and relations $\sin \epsilon_{n} \mu^{+}(\pi) \approx$ $\epsilon_{n} \mu^{+}(\pi), \cos \epsilon_{n} \mu^{+}(\pi) \approx 1$, integrating by parts and using the properties of the kernel $A(x, t)$, we have

$$
\begin{aligned}
\epsilon_{n} \approx & \frac{1}{\lambda_{n}^{0} \dot{\Delta}\left(\lambda_{n}^{0}\right)}\left\{A\left(\pi, \mu^{+}(\pi)\right) \frac{\cos \left(\lambda_{n}^{0}+\epsilon_{n}\right)}{\lambda_{n}^{0}+\epsilon_{n}} \mu^{+}(\pi)\right\} \\
& -\frac{1}{\lambda_{n}^{0} \dot{\Delta}\left(\lambda_{n}^{0}\right)}\left\{\left[A\left(\pi, \mu^{-}(\pi)+0\right)-A\left(\pi, \mu^{-}(\pi)+0\right)\right] \frac{\cos \left(\lambda_{n}^{0}+\epsilon_{n}\right)}{\lambda_{n}^{0}+\epsilon_{n}} \mu^{-}(\pi)\right\} \\
& -\frac{1}{\lambda_{n}^{0} \dot{\Delta}\left(\lambda_{n}^{0}\right)}\left\{\int_{0}^{\mu^{+}(\pi)} A_{t}(\pi, t) \frac{\cos \left(\lambda_{n}^{0}+\epsilon_{n}\right)}{\lambda_{n}^{0}+\epsilon_{n}} t d t\right\} \\
= & \frac{d_{n}}{\lambda_{n}^{0}+\epsilon_{n}}+\frac{\tilde{\eta}_{n}}{\lambda_{n}^{0}+\epsilon_{n}},
\end{aligned}
$$

where

$$
\left\{\tilde{\eta}_{n}\right\}:=\left\{\int_{0}^{\mu^{+}(\pi)} A_{t}(\pi, t) \cos \left(\lambda_{n}^{0}+\epsilon_{n}\right) t d t\right\} .
$$

Let us show that $\left\{\tilde{\eta}_{n}\right\} \in l_{2}$. It is obvious that

$$
\int_{0}^{\mu^{+}(\pi)} A_{t}(\pi, t) \cos \lambda t d t
$$

can be reduced to

$$
\int_{-\mu^{+}(\pi)}^{\mu^{+}(\pi)} R(t) e^{i \lambda t} d t
$$

where $R(t) \in L_{2}\left(-\mu^{+}(\pi), \mu^{+}(\pi)\right)$. Now, take

$$
\zeta(\lambda):=\int_{-\mu^{+}(\pi)}^{\mu^{+}(\pi)} R(t) e^{i \lambda t} d t
$$


It is clear from [19] (p.66) that $\left\{\zeta_{n}\right\}=\left\{\zeta\left(\lambda_{n}\right)\right\} \in l_{2}$. By virtue of this we have $\tilde{\eta}_{n} \in l_{2}$ (see [20, 21]). Therefore, as

$$
\eta_{n}=\frac{\tilde{\eta}_{n}}{\lambda_{n}^{0}+\epsilon_{n}}
$$

the validity of $\eta_{n} \in l_{2}$ can be seen directly. Lemma is proved.

\section{Expansion formula}

Assume that $\lambda^{2}$ is not a spectrum point of operator $A$. Then, there exists resolvent operator $R_{\lambda^{2}}(A)=\left(A-\lambda^{2} I\right)^{-1}$. Let us find the expression of the operator $R_{\lambda^{2}}(A)$.

Lemma 8 The resolvent $R_{\lambda^{2}}(A)$ is the integral operator with the kernel

$$
G(x, t ; \lambda)=-\frac{1}{\Delta(\lambda)} \begin{cases}\varphi(t, \lambda) \psi(x, \lambda), & t \leq x, \\ \psi(t, \lambda) \varphi(x, \lambda), & t \geq x .\end{cases}
$$

Proof To construct the resolvent operator of $A$, we need to solve the boundary value problem

$$
\begin{aligned}
& -y^{\prime \prime}+q(x) y=\lambda^{2} \rho(x) y+\rho(x) f(x), \\
& y(0)=0, \\
& \lambda^{2}\left[\beta_{1} y^{\prime}(\pi)+\beta_{2} y(\pi)\right]+\alpha_{1} y(\pi)+\alpha_{2} y^{\prime}(\pi)=f_{2},
\end{aligned}
$$

where $f(x) \in D(A)$. By applying the method of variation of constants, we seek the solution of the problem (20)-(22) in the following form

$$
y(x, \lambda)=c_{1}(x, \lambda) \psi(x, \lambda)+c_{2}(x, \lambda) \varphi(x, \lambda),
$$

and we get the coefficients $c_{1}(x, \lambda)$ and $c_{2}(x, \lambda)$ as

$$
\begin{aligned}
& c_{1}(x, \lambda)=c_{1}(0, \lambda)-\frac{1}{\Delta(\lambda)} \int_{0}^{x} \varphi(t, \lambda) f(t) \rho(t) d t, \\
& c_{2}(x, \lambda)=-\frac{1}{\Delta(\lambda)} \int_{x}^{\pi} \psi(t, \lambda) f(t) \rho(t) d t+c_{2}(\pi, \lambda) .
\end{aligned}
$$

Substituting (24) and (25) into (23) and taking into account the boundary conditions (21) and (22), we have

$$
y(x, \lambda)=\int_{0}^{\pi} G(x, t ; \lambda) f(t) \rho(t) d t-\frac{f_{2}}{\Delta(\lambda)} \varphi(x, \lambda),
$$

where $G(x, t ; \lambda)$ is as in $(19)$.

Theorem 9 The eigenfunctions $\varphi\left(x, \lambda_{n}\right)_{n \geq 1}$ of the boundary value problem (1)-(3) form a complete system in $L_{2, \rho}(0, \pi) \oplus \mathbb{C}$. 
Proof With the help of (9) and (11), we can write

$$
\psi\left(x, \lambda_{n}\right)=\frac{\dot{\Delta}\left(\lambda_{n}\right)}{2 \lambda_{n} \gamma_{n}} \varphi\left(x, \lambda_{n}\right)
$$

Using (19) and (26), we get

$$
\operatorname{Res}_{\lambda=\lambda_{n}} y(x, \lambda)=-\frac{1}{2 \lambda_{n} \gamma_{n}} \varphi\left(x, \lambda_{n}\right)\left\{\int_{0}^{\pi} \varphi\left(t, \lambda_{n}\right) f(t) \rho(t) d t+\frac{f_{2}}{k_{n}}\right\} .
$$

Now, let $f(x) \in L_{2, \rho}(0, \pi) \oplus \mathbb{C}$ and assume

$$
\left(\varphi\left(x, \lambda_{n}\right), f(x)\right)=\int_{0}^{\pi} \varphi\left(x, \lambda_{n}\right) \overline{f_{1}(x)} \rho(x) d x+\frac{f_{2}\left(\beta_{1} \varphi^{\prime}\left(\pi, \lambda_{n}\right)+\beta_{2} \varphi\left(\pi, \lambda_{n}\right)\right)}{\chi}=0 .
$$

Then from (28), we have $\operatorname{Res}_{\lambda=\lambda_{n}} y(x, \lambda)=0$. Consequently, for fixed $x \in[0, \pi]$ the function $y(x, \lambda)$ is entire with respect to $\lambda$.

Let us denote that

$$
G_{\delta}:=\left\{\lambda:\left|\lambda-\lambda_{n}^{0}\right| \geq \delta, n=0, \mp 1, \mp 2, \ldots\right\},
$$

where $\delta$ is a sufficiently small positive number. (16) is valid from Theorem 12.4 in [22] for $\lambda \in G_{\delta}$.

On the other hand, we can say from Lemma 1.3.1 in [19] that for every $f(x) \in L_{1}(0, \pi)$, the following relation holds

$$
\begin{aligned}
& \lim _{|\lambda| \rightarrow \infty} \max _{0 \leq x \leq \pi}\left\{e^{-|\operatorname{Im} \lambda| x \mid}\left|\int_{0}^{x} f(t) \cos \lambda t d t\right|\right\} \\
& =\lim _{|\lambda| \rightarrow \infty} \max _{0 \leq x \leq \pi}\left\{e^{-|\operatorname{Im} \lambda| x}\left|\int_{0}^{x} f(t) \sin \lambda t d t\right|\right\}=0 .
\end{aligned}
$$

Also, for $|\lambda| \rightarrow \infty$, the relations below hold

$$
\begin{aligned}
& \varphi(x, \lambda)=O\left(\frac{1}{|\lambda|} e^{|\operatorname{Im} \lambda| \mu^{+}(x)}\right), \\
& \varphi^{\prime}(x, \lambda)=\varphi_{0}^{\prime}(x, \lambda)+O\left(\frac{1}{|\lambda|} e^{|\operatorname{Im} \lambda| \mu^{+}(x)}\right)=O\left(e^{|\operatorname{Im} \lambda| \mu^{+}(x)}\right), \\
& \psi(x, \lambda)=O\left(\frac{1}{|\lambda|} e^{|\operatorname{Im} \lambda|\left(\mu^{+}(\pi)-\mu^{+}(x)\right)}\right), \\
& \psi^{\prime}(x, \lambda)=\psi_{0}^{\prime}(x, \lambda) O\left(\frac{1}{|\lambda|} e^{|\operatorname{Im} \lambda|\left(\mu^{+}(\pi)-\mu^{+}(x)\right)}\right)=O\left(e^{|\operatorname{Im} \lambda|\left(\mu^{+}(\pi)-\mu^{+}(x)\right)}\right) .
\end{aligned}
$$

From the estimates (31)-(34), it is obvious that

$$
|\Delta(\lambda)| \geq C_{\delta} \frac{1}{|\lambda|} e^{|\operatorname{Im} \lambda| \mu^{+}(\pi)}, \quad \lambda \in G_{\delta} .
$$

From (26), it follows that for fixed $\delta>0$ and sufficiently large $\lambda^{*}>0$, we have

$$
|y(x, \lambda)| \leq \frac{C_{\delta}}{|\lambda|}, \quad \lambda \in G_{\delta},|\lambda| \geq \lambda^{*}
$$


Using maximum principle for module of analytic functions and Liouville theorem, we get $y(x, \lambda) \equiv 0$. From this and the expression of the boundary value problem (20)-(22), we obtain that $f(x) \equiv 0$ a.e. on $[0, \pi]$. Thus, we reach the completeness of the eigenfunctions $\varphi\left(x, \lambda_{n}\right)$ in $L_{2, \rho}(0, \pi) \oplus \mathbb{C}$.

Theorem 10 If $f(x) \in D(A)$, then the expansion formula

$$
f(x)=\sum_{n=1}^{\infty} a_{n} \varphi\left(x, \lambda_{n}\right)
$$

is valid, where

$$
a_{n}=\frac{1}{2 \gamma_{n}} \int_{0}^{\pi} \varphi\left(t, \lambda_{n}\right) f(t) \rho(t) d t
$$

and the series converges uniformly with respect to $x \in[0, \pi]$. For $f(x) \in L_{2, \rho}(0, \pi)$, the series converges in $L_{2, \rho}(0, \pi)$, moreover, the Parseval equality holds

$$
\int_{0}^{\pi}|f(x)|^{2} \rho(x) d x=\sum_{n=1}^{\infty} \gamma_{n}\left|a_{n}\right|^{2}
$$

Proof Since $\varphi(x, \lambda)$ and $\psi(x, \lambda)$ are the solutions of the boundary value problem (1)-(3), we have

$$
\begin{aligned}
y(x, \lambda)= & -\frac{1}{\lambda^{2} \Delta(\lambda)}\left\{\psi(x, \lambda) \int_{0}^{x}\left[-\varphi^{\prime \prime}(t, \lambda)+q(t) \varphi(t, \lambda)\right] f(t) d t\right\} \\
& -\frac{1}{\lambda^{2} \Delta(\lambda)}\left\{\varphi(x, \lambda) \int_{x}^{\pi}\left[-\psi^{\prime \prime}(t, \lambda)+q(t) \psi(t, \lambda)\right] f(t) d t\right\} \\
& -\frac{f_{2}}{\Delta(\lambda)} \varphi(x, \lambda) .
\end{aligned}
$$

Integrating by parts and taking into account the boundary conditions (2), (3), we obtain

$$
y(x, \lambda)=-\frac{1}{\lambda^{2}} f(x)-\frac{1}{\lambda^{2}}\left\{Z_{1}(x, \lambda)+Z_{2}(x, \lambda)\right\}-\frac{f_{2}}{\Delta(\lambda)} \varphi(x, \lambda),
$$

where

$$
\begin{aligned}
Z_{1}(x, \lambda)= & \frac{1}{\Delta(\lambda)}\left\{\psi(x, \lambda) \int_{0}^{x} \varphi^{\prime}(t, \lambda) g(t) d t+\varphi(x, \lambda) \int_{x}^{\pi} \psi^{\prime}(t, \lambda) g(t) d t\right\}, \\
Z_{2}(x, \lambda)= & \frac{1}{\Delta(\lambda)}\left\{\psi(x, \lambda) \int_{0}^{x} \varphi(t, \lambda) q(t) f(t) d t\right\} \\
& +\frac{1}{\Delta(\lambda)}\left\{\varphi(x, \lambda) \int_{x}^{\pi} \psi(t, \lambda) q(t) f(t) d t+\varphi(x, \lambda) \psi^{\prime}(\pi, \lambda) f(\pi)\right\},
\end{aligned}
$$

as $g(t)=f^{\prime}(t)$. Let us consider the following contour integral

$$
I_{n}(x)=\frac{1}{2 \pi i} \oint_{\Gamma_{n}} \lambda y(x, \lambda) d \lambda,
$$


where $\Gamma_{n}=\left\{\lambda:|\lambda|=\left|\lambda_{n}^{0}\right|+\frac{\tau}{2}\right\}$ is a contour oriented counter-clockwise, and $n$ is a sufficiently large natural number. With the help of Residue theorem, we get

$$
I_{n}(x)=2 \sum_{n=1}^{\infty} \operatorname{Res}_{\lambda=\lambda_{n}}[\lambda y(x, \lambda)]=\sum_{n=1}^{\infty} a_{n} \varphi\left(x, \lambda_{n}\right)+\sum_{n=1}^{\infty} \frac{\lambda f_{2}}{\dot{\Delta}\left(\lambda_{n}\right)} \varphi\left(x, \lambda_{n}\right),
$$

where

$$
a_{n}=\frac{1}{\gamma_{n}} \int_{0}^{\pi} \varphi\left(t, \lambda_{n}\right) f(t) \rho(t) d t .
$$

On the other hand, taking into account (38), we have

$$
I_{n}(x)=-f(x)+\frac{1}{2 \pi i} \oint_{\Gamma_{n}}\left(Z_{1}(x, \lambda)+Z_{2}(x, \lambda)\right) d \lambda .
$$

Comparing (39) and (40), we obtain

$$
\sum_{n=1}^{\infty} a_{n} \varphi\left(x, \lambda_{n}\right)+\sum_{n=1}^{\infty} \frac{\lambda f_{2}}{\dot{\Delta}\left(\lambda_{n}\right)} \varphi\left(x, \lambda_{n}\right)=-f(x)+\epsilon_{n}(x),
$$

where

$$
\epsilon_{n}(x)=-\frac{1}{2 \pi i} \int_{\Gamma_{n}}\left(Z_{1}(x, \lambda)+Z_{2}(x, \lambda)\right) d \lambda .
$$

Thus, we obtain

$$
f(x)=\sum_{n=1}^{\infty} \frac{1}{\gamma_{n}} \varphi\left(x, \lambda_{n}\right)\left\{\int_{0}^{\pi} \varphi\left(t, \lambda_{n}\right) f(t) \rho(t) d t\right\}+\epsilon_{n}(x) .
$$

Now, let us show that

$$
\lim _{n \rightarrow \infty} \max _{x \in[0, \pi]}\left|\epsilon_{n}(x)\right|=0
$$

From estimates (31)-(34) of solutions $\varphi(x, \lambda), \psi(x, \lambda)$ and the inequality (35) for the function $\Delta(\lambda)$, it follows, for fixed $\delta>0$ and sufficiently large $\lambda^{*}>0$

$$
\max _{x \in[0, \pi]}\left|Z_{2}(x, \lambda)\right| \leq \frac{C_{2}}{|\lambda|}, \quad \lambda \in G_{\delta},|\lambda| \geq \lambda^{* \prime} .
$$

Let us show that $\lim _{|\lambda| \rightarrow \infty} \max _{0 \leq x \leq \pi}\left|Z_{1}(x, \lambda)\right|=0$. If we suppose that $g(t) \in A C[0, \pi]$, and by then integrate by parts the expression of $Z_{1}(x, \lambda)$, we obtain

$$
Z_{1}(x, \lambda)=-\frac{1}{\Delta(\lambda)}\left\{\psi(x, \lambda) \int_{0}^{x} \varphi(t, \lambda) g^{\prime}(t) d t+\varphi(x, \lambda) \int_{x}^{\pi} \psi(t, \lambda) g^{\prime}(t) d t\right\} .
$$

Hence, similar to $Z_{2}(x, \lambda)$, we have

$$
\max _{x \in[0, \pi]}\left|Z_{1}(x, \lambda)\right| \leq \frac{C_{1}}{|\lambda|}, \quad \lambda \in G_{\delta},|\lambda| \geq \lambda^{*} .
$$


In general case, let us take an arbitrary fixed number $\epsilon>0$ and assume that $g_{\epsilon}(t) \epsilon$ $A C[0, \pi]$, such that $\int_{0}^{\pi}\left|g(t)-g_{\epsilon}(t)\right| d t<\epsilon$. Then we can find a $\lambda^{* * *}$ that for $\lambda \in G_{\delta}$ and $|\lambda|>\lambda^{* *}$. Also, using the equation below,

$$
\begin{aligned}
Z_{1}(x, \lambda)= & \frac{1}{\Delta(\lambda)}\left\{\psi(x, \lambda) \int_{0}^{x}\left(g(t)-g_{\epsilon}(t)\right) \varphi^{\prime}(t, \lambda) d t\right\} \\
& +\frac{1}{\Delta(\lambda)}\left\{\varphi(x, \lambda) \int_{x}^{\pi}\left(g(t)-g_{\epsilon}(t)\right) \psi^{\prime}(t, \lambda) d t\right\} \\
& +\frac{1}{\Delta(\lambda)}\left\{\psi(x, \lambda) \int_{0}^{x}\left(g_{\epsilon}(t)\right) \varphi^{\prime}(t, \lambda) d t+\varphi(x, \lambda) \int_{x}^{\pi}\left(g_{\epsilon}(t)\right) \psi^{\prime}(t, \lambda) d t\right\},
\end{aligned}
$$

and with the help of the estimates of functions $\varphi(x, \lambda), \psi(x, \lambda)$ and $\Delta(\lambda)$, we get

$$
\max _{x \in[0, \pi]}\left|Z_{1}(x, \lambda)\right| \leq C \int_{0}^{\pi}\left|g(t)-g_{\epsilon}(t)\right| d t+\frac{\tilde{C}(\epsilon)}{|\lambda|}<C \cdot \epsilon+\frac{\tilde{C}(\epsilon)}{|\lambda|},
$$

as $\lambda \in G_{\delta},|\lambda| \geq \lambda^{* * *}$. Hence we have

$$
\varlimsup_{|\lambda \rightarrow \infty|} \max \left|Z_{1}(x, \lambda)\right| \leq C \cdot \epsilon \quad\left(\lambda \in G_{\delta}\right) .
$$

From the arbitrariness of $\epsilon$, we reach

$$
\lim _{|\lambda| \rightarrow \infty} \max _{x \in[0, \pi]}\left|Z_{1}(x, \lambda)\right|=0, \quad \lambda \in G_{\delta}
$$

The validity of (42) can be easily seen from (43) and (44). Thus, we obtain

$$
f(x)=\sum_{n=1}^{\infty} \frac{1}{\gamma_{n}} \varphi\left(x, \lambda_{n}\right)\left\{\int_{0}^{\pi} \varphi\left(t, \lambda_{n}\right) f(t) \rho(t) d t\right\}
$$

If we take

$$
a_{n}=\frac{1}{\gamma_{n}} \int_{0}^{\pi} \varphi\left(t, \lambda_{n}\right) f(t) \rho(t) d t
$$

the last equation gives us the expansion formula

$$
f(x)=\sum_{n=1}^{\infty} a_{n} \varphi\left(x, \lambda_{n}\right) .
$$

Since the system of $\varphi\left(x, \lambda_{n}\right)_{n \geq 1}$ is complete and orthogonal in $L_{2, \rho}(0, \pi) \oplus \mathbb{C}$, the Parseval equality

$$
\int_{0}^{\pi}|f(x)|^{2} \rho(x) d x=\sum_{n=1}^{\infty} \gamma_{n}\left|a_{n}\right|^{2}
$$

holds. Extension of the Parseval equality to an arbitrary vector-function of the class $L_{2}(a, b)$ can be carried out by usual methods. 


\section{Weyl solution, Weyl function}

Let $\Phi(x, \lambda)$ be the solution of equation (1) that satisfies the conditions

$$
\begin{aligned}
& \Phi(0, \lambda)=1, \\
& \lambda^{2}\left[\beta_{1} \Phi^{\prime}(\pi, \lambda)+\beta_{2} \Phi(\pi, \lambda)\right]+\alpha_{1} \Phi(\pi, \lambda)+\alpha_{2} \Phi^{\prime}(\pi, \lambda)=0 .
\end{aligned}
$$

Denote by $c(x, \lambda)$ the solution of equation (1), which satisfies the initial conditions $c(0, \lambda)=1, c^{\prime}(0, \lambda)=0$. Then the solution $\psi(x, \lambda)$ can be represented as follows

$$
\psi(x, \lambda)=\psi^{\prime}(0, \lambda) \varphi(x, \lambda)-\Delta(\lambda) c(x, \lambda)
$$

or

$$
-\frac{\psi(x, \lambda)}{\Delta(\lambda)}=c(x, \lambda)-\frac{\psi^{\prime}(0, \lambda)}{\Delta(\lambda)} \varphi(x, \lambda) .
$$

Denote

$$
M(\lambda):=-\frac{\psi(0, \lambda)}{\Delta(\lambda)} .
$$

It is clear that

$$
\Phi(x, \lambda)=c(x, \lambda)+M(\lambda) \varphi(x, \lambda) .
$$

The functions $\Phi(x, \lambda)$ and $M(\lambda)=\Phi^{\prime}(0, \lambda)$ are respectively called the Weyl solution and the Weyl function of the boundary value problem (1)-(3). The Weyl function is a meromorphic function having simple poles at points $\lambda_{n}$ eigenvalues of boundary value problem (1)-(3). Relations (46), (48) yield

$$
\Phi(x, \lambda)=-\frac{\psi(x, \lambda)}{\Delta(\lambda)}
$$

It can be shown that

$$
\langle\Phi(x, \lambda), \varphi(x, \lambda)\rangle \equiv 1
$$

Let us take into consideration a boundary value problem with the coefficient $\tilde{q}(x)$ similar to (1)-(3) and assume that if an element $\alpha$ belongs to boundary value problem (1)-(3), then $\tilde{\alpha}$ belongs to one with $\tilde{q}(x)$.

Validity of the equation below can be shown analogously to [8]

$$
M(\lambda)=M(0)+\sum_{n=1}^{\infty} \frac{\lambda^{2}}{\gamma_{n} \lambda_{n}^{2}\left(\lambda^{2}-\lambda_{n}^{2}\right)} .
$$

Theorem 11 The boundary value problem (1)-(3) is identically denoted by the Weyl function $M(\lambda) .($ If $M(\lambda)=\tilde{M}(\lambda)$, then $q(x)=\tilde{q}(x)$.) 
Proof Let us identify the matrix $P(x, \lambda)=\left[P_{j k}(x, \lambda)\right]_{j, k=1,2}$ as

$$
P(x, \lambda)\left(\begin{array}{ll}
\tilde{\varphi}(x, \lambda) & \tilde{\Phi}(x, \lambda) \\
\tilde{\varphi}^{\prime}(x, \lambda) & \tilde{\Phi}^{\prime}(x, \lambda)
\end{array}\right)=\left(\begin{array}{cc}
\varphi(x, \lambda) & \Phi(x, \lambda) \\
\varphi^{\prime}(x, \lambda) & \Phi^{\prime}(x, \lambda)
\end{array}\right) .
$$

From (50) and (52), we have

$$
\begin{aligned}
& \varphi(x, \lambda)=P_{11}(x, \lambda) \tilde{\varphi}(x, \lambda)+P_{12}(x, \lambda) \tilde{\varphi}^{\prime}(x, \lambda), \\
& \Phi(x, \lambda)=P_{11}(x, \lambda) \tilde{\Phi}(x, \lambda)+P_{12}(x, \lambda) \tilde{\Phi}^{\prime}(x, \lambda),
\end{aligned}
$$

or

$$
\begin{aligned}
& P_{11}(x, \lambda)=\tilde{\varphi}^{\prime}(x, \lambda) \Phi(x, \lambda)-\varphi^{\prime}(x, \lambda) \tilde{\Phi}^{\prime}(x, \lambda), \\
& P_{12}(x, \lambda)=\varphi(x, \lambda) \tilde{\Phi}^{\prime}(x, \lambda)-\tilde{\varphi}(x, \lambda) \Phi(x, \lambda) .
\end{aligned}
$$

Taking equation (49) into consideration in (54), we get

$$
\begin{aligned}
& P_{11}(x, \lambda)=1+\frac{1}{\Delta(\lambda)} \psi(x, \lambda)\left(\varphi^{\prime}(x, \lambda)-\tilde{\varphi}(x, \lambda)\right)+\frac{1}{\Delta(\lambda)} \varphi(x, \lambda)\left(\tilde{\psi}^{\prime}(x, \lambda)-\psi(x, \lambda)\right), \\
& P_{12}(x, \lambda)=\frac{1}{\Delta(\lambda)}(-\varphi(x, \lambda) \tilde{\psi}(x, \lambda)+\tilde{\varphi}(x, \lambda) \psi(x, \lambda)) .
\end{aligned}
$$

Now, from the estimates

$$
\left|\varphi^{\prime}(x, \lambda)-\tilde{\varphi}(x, \lambda)\right|=O\left(\frac{1}{|\lambda|} e^{|\operatorname{Im} \lambda| \mu^{+}(x)}\right), \quad|\lambda| \rightarrow \infty,
$$

and

$$
\left|\psi^{\prime}(x, \lambda)-\tilde{\psi}(x, \lambda)\right|=O\left(\frac{1}{|\lambda|} e^{|\operatorname{Im} \lambda|\left(\mu^{+}(\pi)-\mu^{+}(x)\right)}\right), \quad|\lambda| \rightarrow \infty
$$

we have from equation (55)

$$
\lim _{|\lambda| \rightarrow \infty} \max _{0 \leq x \leq \pi}\left|P_{11}(x, \lambda)-1\right|=\lim _{|\lambda| \rightarrow \infty} \max _{0 \leq x \leq \pi}\left|P_{12}(x, \lambda)\right|=0
$$

for $\lambda \in G_{\delta}$. Now, if we take consideration equation (48) into (53), we get

$$
\begin{aligned}
& P_{11}(x, \lambda)=\tilde{\varphi}^{\prime}(x, \lambda) c(x, \lambda)-\varphi(x, \lambda) \tilde{c}^{\prime}(x, \lambda)+\tilde{\varphi}^{\prime}(x, \lambda) \varphi(x, \lambda)[M(\lambda)-\tilde{M}(\lambda)], \\
& P_{12}(x, \lambda)=\varphi(x, \lambda)-\tilde{c}(x, \lambda)-\tilde{\varphi}(x, \lambda) c^{\prime}(x, \lambda)+\varphi(x, \lambda) \tilde{\varphi}(x, \lambda)[\tilde{M}(\lambda)-M(\lambda)] .
\end{aligned}
$$

Therefore, if $M(\lambda)=\tilde{M}(\lambda)$, then $P_{11}(x, \lambda)$ and $P_{12}(x, \lambda)$ are entire functions for every fixed $x$. It can easily be seen from equation (56) that $P_{11}(x, \lambda)=1$ and $P_{12}(x, \lambda)=0$. Consequently, we get $\varphi(x, \lambda) \equiv \tilde{\varphi}(x, \lambda)$ and $\Phi(x, \lambda) \equiv \tilde{\Phi}(x, \lambda)$ for every $x$ and $\lambda$. Hence, we arrive at $q(x) \equiv$ $\tilde{q}(x)$.

Theorem 12 The spectral data identically define the boundary value problem (1)-(3). 
Proof From (51), it is clear that the function $M(\lambda)$ can be constructed by $\lambda_{n}$. Since $\lambda_{n}=\tilde{\lambda}_{n}$ for every $n \in \mathbb{N}$, from Theorem 10 , we can say that $M(\lambda)=\tilde{M}(\lambda)$. Then from Theorem 11 , it is obvious that $A=\tilde{A}$.

\section{Competing interests}

The authors declare that they have no competing interests.

Authors' contributions

All authors contributed equally to the manuscript and read and approved the final manuscript.

\section{Acknowledgements}

This work is supported by the Scientific and Technological Research Council of Turkey (TÜBITAK)

Received: 5 April 2013 Accepted: 29 July 2013 Published: 14 August 2013

\section{References}

1. Fulton, СT: Two-point boundary-value problems with eigenvalue parameter contained in the boundary conditions. Proc. R. Soc. Edinb. 77, 293-308 (1977)

2. Tikhonov, AN, Samarskii, AA: Equations of Mathematical Physics. Dover Books on Physics and Chemistry. Dover, New York (1990)

3. Kapustin, NY, Moisseev, El: On a spectral problem with spectral parameter in boundary condition. Differ. Equ. 33 , 115-119 (1997)

4. Benedek, A, Panzone, R: On inverse eigenvalue problem for a second order differential equation with parameter contained in the boundary conditions. Notas Algebra Analysis 9, 1-13 (1980)

5. Mamedov, SG: Determination of a second order differential equation with respect to two spectra with a spectral parameter entering into the boundary conditions (Russian). Izv. Akad. Nauk Azerb. SSR, Ser. Fiz.-Tekh. Mat. Nauk 3 , 15-22 (1982)

6. Browne, PJ, Sleeman, BD: Inverse nodal problems for Sturm-Liouville equations with eigenparameter dependent boundary conditions. Inverse Probl. 12(4), 377-381 (1996)

7. Binding, PA, Browne, PJ, Watson, BA: Inverse spectral problems for Sturm-Liouville equations with eigenparameter dependent boundary conditions. J. Lond. Math. Soc. 62(1), 161-182 (2000)

8. Yurko, VA: Method of Spectral Mapping in the Inverse Problem Theory. VSP, Utrecht (2002)

9. McCarthy, CM, Rundell, W: Eigenparameter dependent inverse Sturm-Liouville problems. Numer. Funct. Anal. Optim. 24(1-2), 85-105 (2003)

10. Guliyev, NJ: Inverse eigenvalue problems for Sturm-Liouville equations with spectral parameter linearly contained in one of the boundary conditions. Inverse Probl. 21(4), 1315-1330 (2005)

11. Amirov, RK, Ozkan, AS, Keskin, B: Inverse problems for impulsive Sturm-Liouville operator with spectral parameter linearly contained in boundary conditions. Integral Transforms Spec. Funct. 20(8), 607-618 (2009)

12. Amirov, RK, Topsakal, N: Inverse problem for Sturm-Liouville operators with Coulomb potential which have discontinuity conditions inside an interval. Math. Phys. Anal. Geom. 13(29), 29-46 (2010)

13. Akhmedova, EN: On representation of solution of Sturm-Liouville equation with discontinuous coefficients. Proc. Inst. Math. Mech. Natl. Acad. Sci. Azerb. XVI(XXIV), 5-9 (2002)

14. Akhmedova, EN: The definition of one class of Sturm-Liouville operators with discontinuous coefficients by Weyl function. Proc. Inst. Math. Mech. Natl. Acad. Sci. Azerb. 22(30), 3-8 (2005)

15. Mamedov, KR: On an inverse scattering problem for a discontinuous Sturm-Liouville equation with a spectral parameter in boundary condition. Bound. Value Probl.. 2010, Article ID 171967 (2010)

16. Mamedov, KR, Kosar, NP: Inverse scattering problem for Sturm-Liouville operator with nonlinear dependence on the spectral parameter in the boundary condition. Math. Methods Appl. Sci. 34(2), 231-241 (2011)

17. Zhdanovich, VF: Formulae for zeros of Dirichlet polynomials and quasipolynomials (Russian). Dokl. Akad. Nauk SSSR $135,5(1960)$

18. Krein, MG, Levin, BY: On entire almost periodic functions of exponential type (Russian). Dokl. Akad. Nauk SSSR 64, 3 (1949)

19. Marchenko, VA: Sturm-Liouville Operators and Their Applications. AMS, Providence (2011)

20. Akhmedova, EN, Huseynov, HM: On eigenvalues and eigenfunctions of one class of Sturm-Liouville operators with discontinuous coefficients. Trans. Acad. Sci. Azerb. Ser. Phys.-Tech. Math. Sci. XXIII(4), 7-18 (2003)

21. Nabiev, AA, Amirov, RK: On the boundary value problem for the Sturm-Liouville equation with the discontinuous coefficient. Math. Methods Appl. Sci. (2012). doi:10.1002/mma.2714

22. Bellman, R, Kuk, KL: Difference-differential equations (Russian). (1967)

doi:10.1186/1687-2770-2013-183

Cite this article as: Mamedov and Cetinkaya: Inverse problem for a class of Sturm-Liouville operator with spectra parameter in boundary condition. Boundary Value Problems 2013 2013:183. 